\title{
A search for planets in the old open cluster NGC $6791^{\star}$
}

\author{
H. Bruntt ${ }^{1}$, F. Grundahl ${ }^{1}$, B. Tingley ${ }^{1}$, S. Frandsen ${ }^{1}$, P. B. Stetson ${ }^{2}$, and B. Thomsen ${ }^{1}$ \\ ${ }^{1}$ Department of Physics and Astronomy, University of Aarhus, Ny Munkegade, 8000 Aarhus C, Denmark \\ 2 Dominion Astrophysical Observatory, Herzberg Institute of Astrophysics, National Research Council, \\ 5071 West Saanich Road, Victoria, British Columbia V9E 2E7, Canada
}

Received 2 May 2003 / Accepted 31 July 2003

\begin{abstract}
We describe the results of a search for transit-like events caused by giant planets occulting stars in the old, metalrich open cluster NGC 6791 based on BVI-colour photometry from eight nights of observations with the $2.54 \mathrm{~m}$ Nordic Optical Telescope. To extract the light curves we have used both PSF photometry (DAOPHOT) and the difference imaging technique (ISIS). We have re-analyzed observations from earlier campaigns to search for multiple transits, determine periods of longperiod variables, and detect eclipsing binaries. We confirm 20 known variables and have discovered 22 new low-amplitude variables with amplitudes in the range 7-40 mmag and periods 1-16 days. We have found the primary and secondary eclipses of two eclipsing binaries based on the new and older photometric campaigns. The search for transit-like events has turned up a few single-transit candidates. The transit depths are $10 \mathrm{mmag}$ in both the $V$ and $I$ filters over periods of 1.0-2.5 hours, but future observations are required to see whether identical transit-like events recur in these same stars.
\end{abstract}

Key words. stars: planetary systems - stars: binaries: eclipsing - stars: variables: general open clusters and associations: individual: NGC 6791

\section{Introduction}

Since the discovery of the first extra-Solar giant planet around 51 Peg (Mayor \& Queloz 1995), around 100 extra-Solar planets have been discovered. Nearly all of these have been found by measuring the variation in radial velocity caused by the orbit of the planet around the parent star. In the case of HD 209458, the planet is known to transit the star causing a reduction of $1.7 \%$ in the light from the star. From the photometric and radial velocity curves Charbonneau et al. (2000) could determine the mass and radius of the planet to within a few percent. Interestingly, the resulting mean density of the planet is quite low due the close vicinity of the parent star (Burrows et al. 2000).

Several attempts have been made to detect extra-Solar planets by monitoring many thousand stars simultaneously with an imaging CCD camera in order to detect the reduction in light due to the transit of a planetary companion. Most notable is the result for 47 Tuc (Gilliland et al. 2000), which failed to detect planets during an 8.3 day search with the Hubble Space Telescope.

Send offprint requests to: $\mathrm{H}$. Bruntt, e-mail: bruntt@phys . au. dk

* Based on observations made with the Nordic Optical Telescope, operated on the island of La Palma jointly by Denmark, Finland, Iceland, Norway, and Sweden, in the Spanish Observatorio del Roque de los Muchachos of the Instituto de Astrofísica de Canarias.
In addition, Udalski et al. (2002a) selected a sample of 52000 stars from the OGLE-III (Wozniak et al. 2002) campaign. These stars have photometry better than $1.5 \%$ and were observed over 32 nights during a period of 45 days. Udalski et al. reported 47 transiting systems of which 42 exhibited multiple transits. An additional 13 objects were later reported by Udalski et al. (2002b), who used a refined search method. In a subsequent paper Dreizler et al. (2002) presented spectra of 16 of the most promising planet transit candidates found by Udalski et al. (2002a). From these spectra Dreizler et al. (2002) could determine the spectral classes of the stars and in turn constrain the mass and radius ratios of the orbiting object and the parent star. Dreizler et al. found that only two of the 16 transiting objects have radii and masses consistent with being giant planets. The other transiting objects are probably brown dwarfs or M-type stars.

Gonzalez et al. (2000) have made spectroscopic studies to determine the metallicities of stars with planets and from a sample of 39 stars they find $<[\mathrm{Fe} / \mathrm{H}]>=0.18 \pm 0.19$. In comparison, the local average metallicity of G-type dwarfs is $[\mathrm{Fe} / \mathrm{H}]=-0.2$ dex (Rocha-Pinto \& Maciel 1996). Laughlin (2002) states that the probability of a star hosting a planet is 10 times higher for stars with high metallicity $([\mathrm{Fe} / \mathrm{H}]>0.2)$ than for less metal-rich objects. One possible reason for the higher metallicity of stars with planets could be that planets are 
more likely to form in a metal-rich environment (see Laughlin 2002 for a discussion). Another possibility (Laughlin 2002) could be the addition of metal-rich material onto the star, which is mixed up in the convective outer layers. The source could be migrating proto-planets and/or planetesimals from the protoplanetary disc.

Laughlin (2000) also examined the correlation between metallicity and mass of stars with giant planets. Among stars with planets there is a tendency for the stars with higher metallicity to also have larger mass. The proposed explanation is the fact that the higher the mass the shallower the outer convection zone will be, hence for the more massive stars the metal-rich material which is added is mixed through a smaller fraction of the star.

Inspired by these interesting results we selected the old metal-rich open cluster NGC 6791 as our target for a search for planet transits. The metallicity of the cluster is a subject of debate. Taylor (2001) states that "the best that can be said at present is that the cluster metallicity lies in the range from +0.16 to +0.44 dex". Two studies published since the Taylor article come to no better agreement: Friel et al. (2002) have derived $[\mathrm{Fe} / \mathrm{H}]=+0.11 \pm 0.010$ from spectroscopy of cluster giants, while Stetson et al. (2003) infer an abundance $\sim+0.3$ by matching the morphology of the cluster color-magnitude diagram to that of recent theoretical isochrones.

In comparison the globular cluster 47 Tuc studied by Gilliland et al. (1999) has $[\mathrm{Fe} / \mathrm{H}]=-0.7$ (Salaris \& Weiss 1998). In this study around 30 transits of short-period giants were expected from the 34000 light curves, i.e. one out of every 1000 stars. The fact that no transits were found is perhaps due to the lower probability of finding planets in a metal-poor system. Another possible explanation is that the relatively high stellar density in a globular cluster may have influenced the evolution of proto-planetary discs or the long-term stability of any planetary systems that may have formed. The dynamical properties of open clusters are not as extreme as for globular clusters and the formation of planets may take place here.

We observed NGC 6791 for $\simeq 8$ hours on each of seven nights and we estimate the probability of detecting a single transit of a close-in planet to be $P_{\text {single }} \simeq 50 \%$ and $P_{\text {twice }} \simeq 7 \%$ for detecting a transit twice for the same star. The probability that a local $\mathrm{G}$ or F-type field star harbours a close-in giant planet is around $0.7 \%$ (Butler et al. 2002) while the geometric probability that the planet will cause a transit is $10 \%$. We have $N_{\sigma<2 \%}=2500$ stars in our sample with light curves with a precision better than $2 \%$ per data point. Assuming that the binary fraction $P_{\text {bin }}$ of the cluster is $15 \%$ the expected number of planets that will be detected is

$N=N_{\sigma<1 \%} * P_{\mathrm{GF}} * P_{\text {geom }} *\left(1-P_{\text {bin }}\right) * P_{\text {single }}=0.8$.

The metallicity of NGC 6791 is significantly higher than the field stars and we may expect to find at least a few candidates with single transits. According to Laughlin (2000) the fraction of stars harbouring planets is $\simeq 10$ times higher for metal-rich stars.

If we detect transits in NGC 6791 and combine this with radial velocity measurements we can put constraints on the mass and radius of the parent star and in turn also constrain the radius of the transiting object from the depth of the transit.

In Sect. 2 we describe the observations and in Sect. 3 we discuss the data reduction. In Sect. 4 we present the calibrated colour-magnitude diagram. In Sect. 5 we discuss the detected transit candidates. In Sects. 6 and 7 we present the new variable stars and the detached eclipsing binaries. Finally, in Sect. 8 we discuss our results and suggest future prospects for planet searches though photometry.

\section{Observations}

The observations for this project were acquired between July 9 and 17, 2001 at the Nordic Optical Telescope (NOT) by one of us (Bruntt). We used the ALFOSC instrument, which gives a field of view of $6^{\prime} 5 \times 6^{\prime} 5$. A $2 \mathrm{k} \times 2 \mathrm{k}$ thinned Loral CCD with a pixel size of $0^{\prime \prime} .188$, read-out noise $6.5 \mathrm{e}^{-}(\mathrm{rms})$, and a gain of $2.5 \mathrm{e}^{-} / \mathrm{ADU}$ was used as detector. The time-series observations were made using $V$ and $I$ filters to be able to distinguish between grey and coloured transits. To first order, planets will cause the same transit depth regardless of colour, while a transit due to a stellar companion or variations due to the star itself (spots) will most likely have different amplitudes in different filters. The typical exposure times used were $180 \mathrm{~s}$ and $90 \mathrm{~s}$ for $V$ and $I$ respectively, chosen such that stars above the turnoff of the cluster at around $V=16$ would be at the saturation limit. In order to reduce the overhead in connection with changing filters we grouped the observations as five exposures in $I$ followed by three in $V$. The read-out time of the detector was $70 \mathrm{~s}$.

A few observations were collected in the $B$ filter as well, to be used for the construction of the colour-magnitude diagram of the cluster.

In order to achieve the highest possible precision in the differential photometry we made small corrective offsets of the telescope during the night to keep the stars in the same position on the detector. To be specific, the $1-\sigma \mathrm{rms}$ scatter of the $x$ and $y$ positions was 2.8 pixels and the median seeing was 5.3 pixels.

Flat fields were obtained with different rotations of the camera (to minimize scattered light effects; see Grundahl \& Sørensen 1996) during evening and morning twilight on each night. A master flat field was constructed by using all flat field images, since no significant night-to-night variations were seen.

On each night we observed several standard fields from Stetson (2000) in order to provide a good transformation to the standard $B V I$ system $^{1}$ (Johnson $B V$ and Cousins $I$ ). The details of this can be found in Stetson et al. (2003). For the remainder of this paper we will refer to the data set from this run as NOT01.

The fundamental parameters of NGC 6791 are given in Table 1.

1 Available from http://cadcwww.hia.nrc.ca/cadcbin/ wdb/astrocat/stetson/query/ 
Table 1. Fundamental properties of NGC 6791: right ascension, declination, age, distance modulus, metallicity, and reddening. The values are taken from Chaboyer et al. (1999) except the metallicity which is from Taylor (2001). Note that the quoted errors are probably too optimistic and do not include systematic errors (except the error on the metallicity).

\begin{tabular}{cccccc}
\hline \hline$\alpha_{2000.0}$ & $\delta_{2000.0}$ & Age [Gyr] & $(m-M)_{V}$ & {$[\mathrm{Fe} / \mathrm{H}]$} & $E(B-V)$ \\
$19^{\mathrm{h}} 20^{\mathrm{m}} 53^{\mathrm{s}}$ & $+37^{\circ} 46^{\prime} 30^{\prime \prime}$ & $8.0(5)$ & $13.37(10)$ & $+0.30(15)$ & $0.10(2)$ \\
\hline
\end{tabular}

Table 2. Log of observations of NGC 6791 from three campaigns. The columns contain the adopted abbreviation of the campaign, the name of the telescope, the duration of the campaign, the number of useful nights $(n)$, the median seeing in the $V$ filter, and the number of images obtained in each of the $B V R I$ filters. The last column gives information on which reduction technique we have used. Note that we have not reduced the $\mathrm{MOCHO1}$ data set, but we have obtained the light curves of the variable stars published by Mochejska et al. (2002).

\begin{tabular}{llrc|c|cccc|c}
\hline \hline & & & & \multicolumn{3}{|c|}{ Number of images } & Reduction \\
Campaign & Telescope & Dates UT & $n$ & {$\left[^{\prime \prime}\right]$} & $N_{B}$ & $N_{V}$ & $N_{R}$ & $N_{I}$ & Method \\
\hline NOT01 & 2.5 m NOT & $9 / 7-17 / 701$ & 7 & $1^{\prime \prime}$. & 58 & 264 & - & 444 & ALLFRAME/ISIS \\
MOCHO1 & 1.2 m FLWO & $6 / 7-1 / 801$ & 18 & $2^{\prime \prime} 1$ & - & 36 & 204 & - & Not Reduced \\
SMR95 & $1.0 \mathrm{~m} \mathrm{JKT}$ & $11 / 7-24 / 795$ & 14 & $1^{\prime \prime} 6$ & - & 601 & - & 153 & ALLFRAME \\
\hline
\end{tabular}

\subsection{Time-series studies of NGC 6791}

Rucinski et al. (1996) carried out a campaign on NGC 6791 for 14 nights in July 1995 to search for detached eclipsing binaries. Despite good weather conditions the noise level in this data set (labelled SMR95 from this point on) is higher compared to the NOTO1 data due to the smaller telescope: the apertures are $1 \mathrm{~m}$ for SMR95 and $2.5 \mathrm{~m}$ for NOTO1. The SMR95 data is still useful for constraining the periods of long-period variables and will be used to search for detached eclipsing binary stars. Rucinski et al. (1996) discovered 11 variable stars (four new ones) and among them were three detached binaries. We refer to Rucinski et al. (1996) for a list of earlier studies on NGC 6791.

A more recent study was made by Mochejska et al. (2002) who have selected NGC 6791 as their first target to search for planetary transits. They have used the FLWO $1.2 \mathrm{~m}$ telescope at Mt. Hopkins, Arizona, from July 6 to August 1, 2001 (we label this data set $\mathrm{MOCHO1}$ ), i.e., partly overlapping with the NOTO1 observations from La Palma. Unfortunately, the weather conditions were quite poor with a median seeing in $V$ of 2." 1 (in contrast, for NOTO1 the median seeing was 1.'0). Mochejska et al. (2002) state that the search for transit-like events still remains to be done, and that a new campaign on the cluster is being planned. Mochejska et al. (2003) have analysed time-series data spanning six years and report the discovery of seven new long-period and irregular variables. Kaluzny (2003) reported the discovery of four new variables in the field.

In Table 2 we give a summary of the observations of NGC 6791 that we have used. For the MOCHO1 data we have only used the light curves of the variable stars from Mochejska et al. (2002); in other words, we have not reduced these data ourselves as we have for NOTO1 and SMR95. Note that the photometric precision of the NOTO1 campaign is superior to the other telescopes, and only these data (seven nights) were used to search for transit-like events.

In addition to the data listed in Table 2 several images taken outside the center of NGC 6791 from NOTO1 and several images from other telescopes were reduced with ALLFRAME as described by Stetson et al. (2003).

\section{Photometry of NGC 6791}

We have used two different methods for obtaining the differential time series photometry: (1) the profile fitting (PSF) approach viz. DAOPHOT/ ALLSTAR/ ALLFRAME (Stetson 1987, 1994) and (2) the image-subtraction method using the IsIs software (Alard \& Lupton 1998). We shall describe our approach for these two methods below.

\subsection{ALLFRAME reductions}

The time-series photometry from SMR95, the new NOTO1 data set, as well as a few frames from other campaigns were reduced using the DAOPHOT/ALLFRAME photometric software (Stetson 1987, 1994). This is described in our companion paper (Stetson et al. 2003). The individual light curves of the variable stars (and the transit-like candidates found from the ISIS light curves) were derived from these data as follows.

Transformation equations relating the instrumental CCD magnitudes to standard-system magnitudes and colours, with corrections for atmospheric extinction, were derived from standard fields observed the same nights as the NGC 6791 observations were obtained. These transformations must then be used again in the reverse sense to convert the observed instrumental magnitudes for the cluster stars to magnitudes on the standard system. Since these transformations are expressed in terms of standard, not instrumental colours, initially each given star was assumed to have a "typical" colour. Then, robust leastsquares methodology was used to find the standard-system magnitude in each of the photometric band-passes which was most consistent with all the observed instrumental magnitudes for that star. These new standard-system magnitudes then implied a new, more realistic colour for the star. These were used for a new least-squares solution for the best standard-system 
magnitudes, and the process was iterated until the derived magnitudes stopped changing. Because the colour terms in the transformations are quite small, with absolute values of well under $0.1 \mathrm{mag} / \mathrm{mag}$, this process converges very quickly. This approach does have the drawback that at each epoch the instrumental magnitude is transformed to a standard-system magnitude based upon the mean colour of each star, averaged over all epochs. In the case of a constant star, this is an optimal approach, but in the case of a variable star it could introduce small systematic errors in the magnitudes observed at the extremes of the star's colour cycle. For a variable star, it would be better to correct each instrumental magnitude according to the star's instantaneous colour, which is possible when light curves are being fitted at the same time as the photometric observations are being calibrated. This was done, for instance, when we used the same software for the Hubble Space Telescope Key Project on the Extra-galactic Distance Scale, where template Cepheid light curves were being fitted to variable-star candidates (see Stetson 1996, 1998). In the present case, we had no preconceived notions as to the forms of individual variable stars' light curves, so we did not try to fit light curves during the photometric reductions in order to be able to predict instantaneous colours; the extraction of light curves was done ex post facto after the calibration of the photometry. Note that this procedure may bias instantaneous standard-system magnitudes by amounts not larger than the colour-transformation terms times half the colour amplitude of a variable star, which will generally lead to a maximum systematic error less than 0.01 mag in the instantaneous magnitudes at the epochs of extreme colour. Systematic errors in the periods, mean magnitudes, and mean colours of the variable stars should be negligible.

\subsection{Difference Imaging with ISIS}

We have used the difference-imaging software ISIS version 2.1 developed by Alard \& Lupton (1998). For each of the filters $V$ and $I$, we selected the images with the best seeing, which were then averaged to generate reference images. For each original image a kernel is computed which describes the variations of the PSF across that image relative to the reference image. The final step is to convolve the reference image by this kernel and subsequently subtract the convolved reference image from each individual image. In principle what will be left in the subtracted images will be the signal that is intrinsically different from the reference image, e.g., variable stars.

One of the advantages of using the difference-image technique is that the signal from variable stars in crowded regions will be less affected by the neighbouring stars, e.g., when compared to profile fitting photometry. Another important point is that variations due to airmass and transparency variations are removed to first order as a part of the image subtraction.

We tried to use the photometry package of ISIS on the subtracted images. Unfortunately, the program produced only meaningless output (all stars were constant). Instead we used the DAOPHOT package to extract aperture photometry in the subtracted image around the position of all stars in the field (found by using ALLSTAR). Normally, DAOPHOT calculates the magnitude of a star as $m=25-2.5 \log (N)$, where $N$ is the number of counts in the stellar profile. But since the number of counts in an aperture can be negative for the difference images, we had to add a short piece of code in the original DAOPHOT program to account for this. In particular, we defined a new "difference magnitude" as $m_{\text {ISIS }}=25$ $2.5 \log \left[\left(N+\sqrt{N_{0}^{2}+N^{2}}\right) / 2\right]$. The zero-point of this magnitude scale is $m_{0}=25-2.5 \log \left(N_{0}\right)$ and we used $N_{0}=1$, i.e., $m=25.0$ when $N=0$. One can show that the number of counts will be $N=N_{0} \sinh \left[0.4 \ln (10)\left(m_{0}-m+2.5 \log (2)\right)\right]$.

We used a range of increasing aperture sizes which were scaled with the seeing (i.e., the measured FWHM) of each image. We then defined magnitude intervals with a width of 1 mag and selected the aperture size to be used within each interval which gave the lowest noise level. Large apertures were used for the bright stars and smaller apertures for fainter stars (the latter will be more affected by sky background noise). Based on growth curves constructed for the direct images (Stetson 1990) we then scaled the counts in each aperture to include all the light.

The relative magnitudes were then computed as $\Delta m=$ $-2.5 \log \left[\left(N_{\text {ref }}-N_{i}\right) / N_{\text {ref }}\right]$, where $N_{\text {ref }}$ is the counts in the reference image and $N_{i}$ is the number of counts in the chosen aperture after applying the correction from the growth curve.

\subsection{Repairing the images}

Any major defects in the images will cause residuals in the subtracted images. There are three major contributors to the defects: cosmic rays, bad columns, and saturated stars, including charge leakage extending down the image columns from the brightest saturated stars. While cosmic rays are normally rejected as part of the ISIS reduction the latter two effects may influence the quality of the image subtraction. In particular the image subtraction gives large residuals around the most saturated stars. We thus decided to repair the saturated pixels in the cores of bright stars and the flux from stars which fall on bad columns. As a result of this procedure, which we will now describe, the residuals in the subtracted images were still present, but only very close to the saturated star.

A short code to replace pixels on the bad columns and the saturated pixels was written in IDL. For each image the code examines the pixels around the brightest stars to look for pixels above the saturation limit (60000 ADU). We also identified stars for which the centroid is within 20 pixels of a bad column. We then computed an "artificial" image where profiles for these stars were inserted using the PSF model found with DAOPHOT for each image, employing for this purpose the positions and instrumental magnitudes found for these stars by ALLSTAR. We then replaced the saturated pixels and the signal on the bad columns in the observed image with the data from the artificial image (including the estimated background level and photometric noise).

We stress that when searching the light curves for transitlike events and variable stars, we clearly mark the points in the light curves where the star is close to a bad column or a 


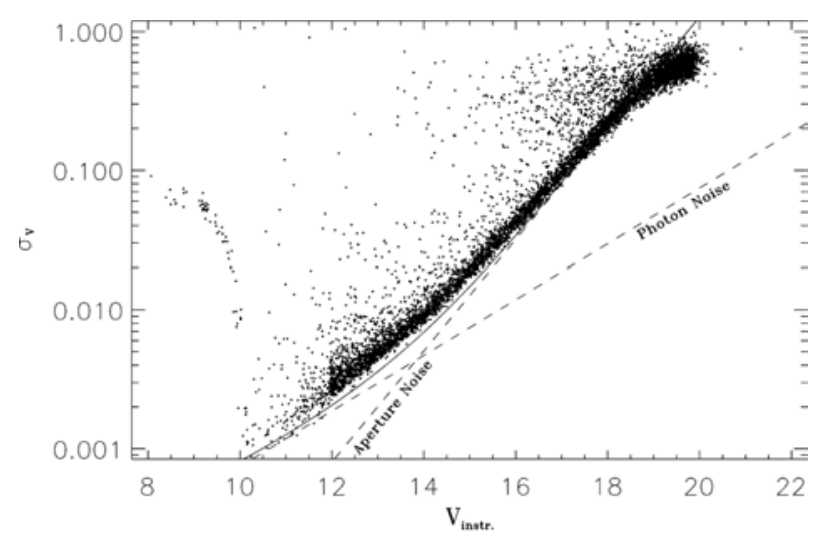

Fig. 1. Measured noise level in $V$ filter (instrumental magnitudes) measured over seven nights from the NOTO1 data set. The reductions were made with ISIS (Alard \& Lupton 1998). The dashed lines mark the photon noise and the aperture noise while the solid line is the quadratic combination of these two.

neighbouring saturated star, since the validity of the measured signal in these cases is indeed questionable.

\subsection{Photometric noise in the IsIs light curves}

In Fig. 1 we plot the image-to-image noise level of the light curves from the NOTO1 data set reduced with ISIS. The dashed lines mark the contribution to the noise from photon statistics and aperture noise (flat field error, sky background determination, and read-out noise) calculated using Eq. (31) in Kjeldsen \& Frandsen (1992):

$\sigma_{\mathrm{ap}}^{2}=\frac{2 \ln 2}{W^{2} \pi e_{\mathrm{ff}}}+\frac{1}{e_{\mathrm{star}}}+\pi r_{\mathrm{ap}}^{2} \frac{e_{\mathrm{sky}}+\sigma_{\mathrm{ccd}}^{2}}{\left(e_{\mathrm{star}}\right)^{2}}$,

where $\sigma_{\text {ap }}^{2}$ is the theoretical total noise level, $W$ is the FWHM of the stellar profile (in pixels), $e_{\mathrm{ff}}$ is the number of electrons in the flat field in one pixel, $e_{\text {star }}$ is the total number of electrons from the star, $r_{\mathrm{ap}}$ is the radius of the aperture (in pixels), $e_{\mathrm{sky}}$ is the number of electrons per pixel in the sky background, and $\sigma_{\text {ccd }}$ is the readout noise in electrons.

The solid line gives the combined theoretical noise level. The brightest stars in the cluster reach a noise level of 1 mmag per point while the turnoff stars $\left(I_{\text {Inst }}=12\right)$ typically have 3 mmag per point. Of the 8455 stars in our sample, 1700 stars have noise $<1 \%$ and for 2500 stars the noise is $<2 \%$ per data point.

The fact that the measured noise level is much higher than the photon noise level for the faint stars shows that the combination of image-subtraction and aperture photometry on the subtracted images is indeed not yet optimal.

The relative transit depth caused by a close-in giant planet is equal to the square of the radius ratio of the planet and the parent star. For stars below the turnoff region $\left(I_{\text {Instr }} \simeq 12\right.$ ), the transit depth of a Jupiter-sized planet will be of the order 13\% (cf. Fig. 2 in Mochejska et al. 2002). Thus the photometric noise limit is well below the limit for detection of transits since we will have several measurements during a transit which is expected to be of the order 1-3 hours.

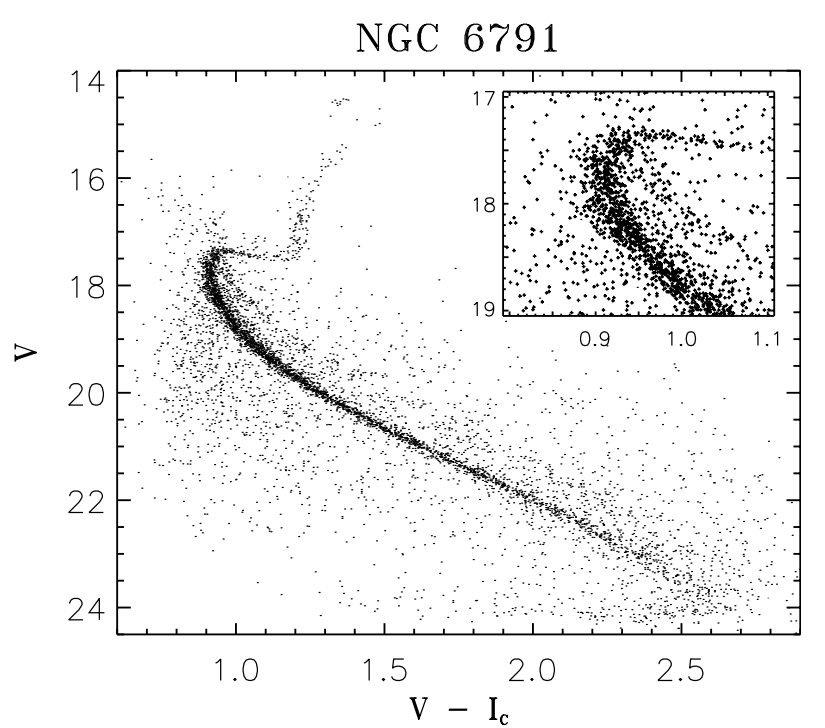

Fig. 2. The calibrated colour-magnitude diagram of NGC 6791. The inserted plot shows the turnoff region: notice the narrow main sequence and the binary sequence.

\section{The calibrated colour-magnitude diagram}

In Fig. 2 we present the colour-magnitude diagram of NGC 6791. It is the result of the ALLFRAME reduction of $958 \mathrm{~V}$ and $737 I$ frames which is described in our companion paper (Stetson et al. 2003). The magnitudes are calibrated to the standard $V$ and $I$ system. The formal error on the turnoff stars is around $0.5 \mathrm{mmag}$ (standard error of the mean) in both $V$ and $I$ for stars around the turnoff while the calibration errors are probably not much greater than 2 mmag. It should be noted, however, that these are repeatability estimates for the magnitudes and colours of individual stars contained within this data set. Stetson et al. present evidence that two stars that appear to have identical photometric properties with one filter/detector combination can differ by up to $20 \mathrm{mmag}$ (standard deviation) when measured with different equipment, in these broad bandpasses. Nevertheless, the calibrated photometry could be very useful as a standard field and is available from the Canadian Astronomy Data Centre (see footnote on p. 324).

\section{The search for transit-like events}

The first seven nights of the NOTO1 campaign were used in the search for transit-like events. The quality of the eighth night was poor by comparison and neglected for that reason. Moreover, the data from the SMR95 campaign was also considered to be too poor for the detection of low-amplitude transits. The NOTO1 light curves were searched for low-amplitude transits using the matched filter technique, first suggested for this task by Jenkins et al. (1996). The decision to use this method to identify transit-like features is based on the work of Tingley (2003), where it is shown that the matched filter performs best. The data from NOTO1 was of very good quality and very consistent, with no variation of the signal-to-noise ratio from night to night. 


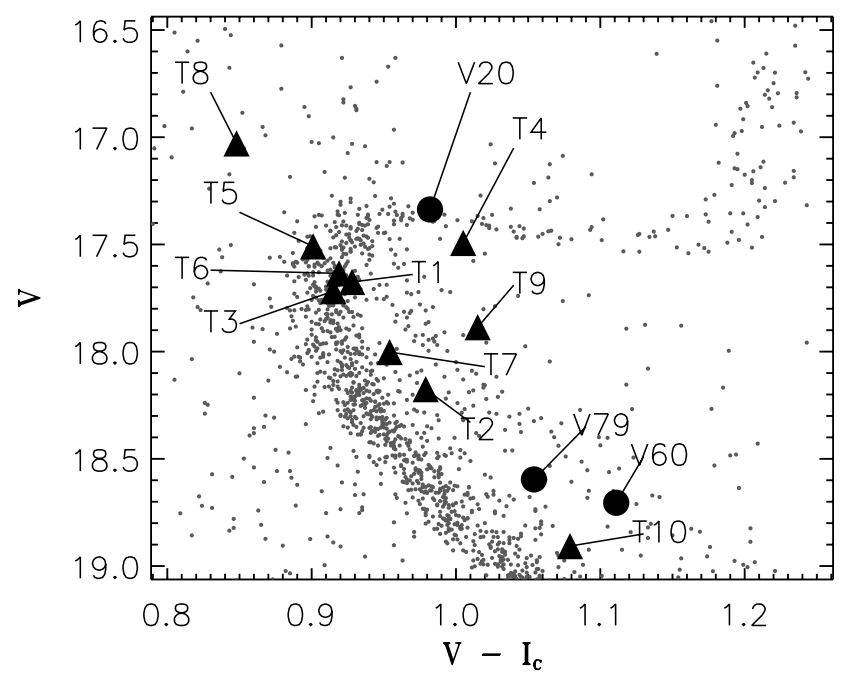

Fig. 3. Colour-magnitude diagram of NGC 6791 with standard $V$ and $V-I_{\text {c }}$ magnitudes from Stetson et al. (2003). The transit candidates (triangles) and the eclipsing binaries (circles) are marked. The stars with the best photometry are also plotted to emphasize the location of the main sequence, the turnoff, and the red giant branch.

Our first implementation of this transit-detection algorithm searched for single transits without using point-to-point noise, which is reasonable considering the consistency of the data. It assumed a square-well transit shape and looked for transits with durations of 2,3 , and 4 hours and depths of 1,2 , and $3 \%$. A histogram was made of the times of the derived events, to determine if any particular epochs were over-producing anomalies, which in theory should be distributed evenly in the case of uncorrelated noise. The epochs that over-produced events were then removed and the analysis repeated. This process was performed several times, which significantly reduced the number of false alarms. Many of the events that remained were also easily identifiable as false alarms, as they could be explained by the contamination of a star by a nearby known variable star, a saturated neighboring star, or by the star being close to the edge of the CCD or a bad column. However, many interesting lowamplitude variable stars were identified, including ones which were not clearly identified by the Stetson $J$-index (cf. Sect. 6).

The second implementation of the transit-detection algorithm searched for multiple transits using point-to-point noise. The point-to-point noise was calculated by choosing a sample of some tens of stars in a relatively narrow magnitude bin with similar, high signal-to-noise ratios. These values were then weighted by the the overall signal-to-noise of the individual light curves. Again using the matched filter with a square-well transit shape, the light curves were searched for repeating transits with periods ranging from 0.8 to 6.3 days, durations of 2 , 3,4 , and 5 hours, and depths of $0.02,0.01$, and 0.005 mag. This implementation identified fewer strong events, but fewer false alarms and variable stars as well, the latter due to the fact that most of them were excluded from the experiment due to their relatively high signal-to-noise ratios.

\subsection{Transit-like light curves}

After rejection of obvious false alarms, we have found ten stars with transit-like events in their light curves. The positions of these stars in the CMD are given in Fig. 3 and their properties are given in Table 3. We have also plotted the stars with the very best photometry, i.e., 3581 stars having the following quality-indices from ALLFRAME: $\mid$ shar $p|<0.25,| \chi-1 \mid<0.25$, $\sigma_{V}<0.04$, and $\sigma_{I}<0.04$. The sharp index measures how well the widths of the PSF and the observed star agree, $\chi$ is essentially the standard goodness-of-fit index, and $\sigma_{V}$ and $\sigma_{I}$ are the estimated rms errors on the PSF magnitudes. We refer to Sect. 4.1 of Stetson et al. (2003) for a detailed discussion of the meanings of these indices.

In Fig. 4 we present portions of the light curves for the ten stars with transit-like light-curve features in the NOTO1 data set. The black points are $V$ measurements while the grey points are $I$ measurements. The times indicated on the plots as $t_{0}$ are HJD-2 452000 for the tick-mark corresponding to 0.0 .

We note that the telescope was offset slightly on the first night at the time $t_{0}=100.52$ to the position which was used for the rest of the NOTO1 campaign. This time coincides with the candidate transits seen in T1, T2, and T3, and with the first of two candidate transits in T10. Similarly, T6, T7, and T8 all have apparent transits around $t_{0}=106.60$, but we note that the durations of these events are generally different. Finally, the observed transit-like event of $\mathrm{T} 2$, is very likely due to the star being close to a bad column on the CCD.

We have only observed single transit-like events except for T6 and T10. However, the durations of the two events seen for T6 are very different. T9 is found to be a long-period lowamplitude variable (V80, cf. Fig. 7), so quite possibly the dip seen at $t_{0}=106.7$ could be the beginning of an eclipse in a binary pair. This seems plausible from the position of $\mathrm{T} 9$ in the CMD in Fig. 3. The transit-like event seen for T4 is very odd, since the $I$ measurements indicate a much longer transit duration than the $V$ measurements.

The most promising cases we have found are $\mathrm{T} 5, \mathrm{~T} 7$, and $\mathrm{T} 8$, for which the transit events have a duration of 1-3 hours. Furthermore, the depths are around $10 \mathrm{mmag}$ in both the $V$ and $I$ filters. These facts are not in contradiction with the expected transit light curves of the short-period giant planets which are known from radial-velocity searches (Butler et al. 2002).

As pointed out by Mallén-Ornelas et al. (2003), the shape of a transit caused by a planet will be characterized by steep ingress/egress slopes and a flat bottom during the transit. The precision of the present photometry is not good enough to see the detailed shape of these shallow events. We would need further data of higher precision to confirm the transits and also to detect multiple transits.

We finally note that the age of NGC 6791 is $8.0 \pm 0.5 \mathrm{Gyr}$ according to Chaboyer et al. (1999) (although we note that Stetson et al. (2003) suggest the age could be significantly greater). Hence the expected rotational periods of the stars at the turnoff would be of the order of weeks, and therefore star spots do not seem like a plausible explanation for the events we have found. 


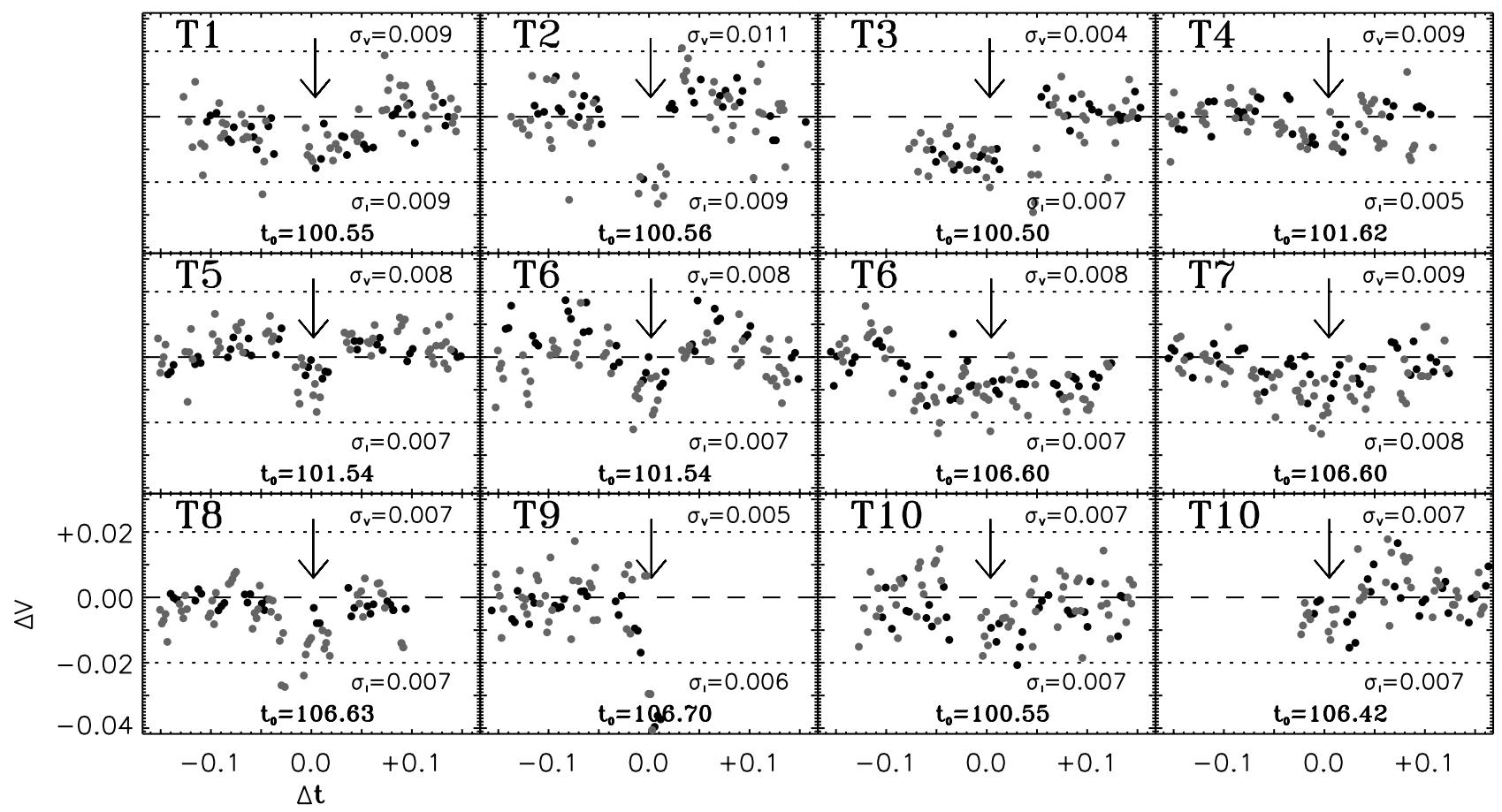

Fig. 4. Light curves of transit candidates. Black and grey symbols are $V$ and $I$ measurements, respectively, and the noise level is given as $\sigma_{V}$ and $\sigma_{I}$. The time $t_{0}$ in each panel corresponds to the time HJD-2452000 of the tick-mark at 0.0. Note that T6 has two transits-like events but of very different duration.

Table 3. We give the name, ID number, $\alpha, \delta, V, V-I$, and $B-I$ for the stars with transit-like light curves. All information except the adapted name in the first column is from Stetson et al. (2003). The positions of the stars in the CMD is shown in Fig. 3 and the light curves can be seen in Fig. 4. As discussed in the text, the transits seen for T1-4, T6, and T9 are not likely to be due to transiting giant planets (they are marked by a * symbol).

\begin{tabular}{|c|c|c|c|c|c|c|}
\hline Name & ID & $\alpha_{2000.0}$ & $\delta_{2000.0}$ & $V$ & $V-I$ & $B-I$ \\
\hline $\mathrm{T} 1^{*}$ & 4891 & $19^{\mathrm{h}} 20^{\mathrm{m}} 43^{\mathrm{s}} \cdot 37$ & $+37^{\circ} 47^{\prime} 31^{\prime \prime} .5$ & 17.675 & 0.928 & 1.818 \\
\hline $\mathrm{T} 2 *$ & 6598 & $19^{\mathrm{h}} 20^{\mathrm{m}} 48^{\mathrm{s}} .65$ & $+37^{\circ} 47^{\prime} 41^{\prime \prime} .1$ & 18.175 & 0.979 & 1.901 \\
\hline T3* & 12616 & $19^{\mathrm{h}} 21^{\mathrm{m}} 07^{\mathrm{s}} .28$ & $+37^{\circ} 47^{\prime} 39^{\prime} 9$ & 17.718 & 0.915 & 1.809 \\
\hline $\mathrm{T} 4^{*}$ & 9049 & $19^{\mathrm{h}} 20^{\mathrm{m}} 55^{\mathrm{s}} .47$ & $+37^{\circ} 44^{\prime} 06^{\prime \prime} 1$ & 17.493 & 1.005 & 1.839 \\
\hline $\mathrm{T} 5$ & 3671 & $19^{\mathrm{h}} 20^{\mathrm{m}} 39^{\mathrm{s}} .07$ & $+37^{\circ} 47^{\prime} 26^{\prime \prime} .1$ & 17.509 & 0.901 & 1.784 \\
\hline $\mathrm{T} 6^{*}$ & 3567 & $19^{\mathrm{h}} 20^{\mathrm{m}} 38^{\mathrm{s}} .63$ & $+37^{\circ} 45^{\prime} 33^{\prime \prime} .3$ & 17.635 & 0.919 & 1.811 \\
\hline $\mathrm{T} 7$ & 3723 & $19^{\mathrm{h}} 20^{\mathrm{m}} 39^{\mathrm{s}} .25$ & $+37^{\circ} 45^{\prime} 39^{\prime} 3$ & 18.002 & 0.954 & 1.827 \\
\hline $\mathrm{T} 8$ & 9020 & $19^{\mathrm{h}} 20^{\mathrm{m}} 55^{\mathrm{s}} .40$ & $+37^{\circ} 47^{\prime} 23^{\prime \prime} .4$ & 17.029 & 0.848 & 1.670 \\
\hline T9* & 12390 & $19^{\mathrm{h}} 21^{\mathrm{m}} 06.48$ & $+37^{\circ} 47^{\prime} 27^{\prime \prime} .8$ & 17.886 & 1.015 & 1.947 \\
\hline $\mathrm{T} 10^{*}$ & 8279 & $19^{\mathrm{h}} 20^{\mathrm{m}} 53^{\mathrm{s}} .42$ & $+37^{\circ} 48^{\prime} 14^{\prime \prime} 6$ & 18.907 & 1.079 & 2.092 \\
\hline
\end{tabular}

To summarize, we cannot claim that what we have observed is indeed caused by a giant planet in any of the cases presented in Fig. 4 and Table 3.

\section{The variable stars in NGC 6791}

To search for variable stars we used the Stetson $J$-index (Stetson 1996). To summarize, the $J$-index is a normalized sum of the deviation of each point (or pair of points with small separations in time) in the light curve compared to the expected noise level for that star. To determine the expected noise level for a given star we used the mean repeatability of a large number of stars with similar magnitude.

In the following we present the phased light curves of some of the variable stars in NGC 6791. In addition to 20 known variables in our field we present 22 new variables. The locations in the CMD of the 22 new variables are shown in Fig. 5. The new variables all have low amplitudes and long periods. This explains why they were not found by Rucinski et al. (1996) or Mochejska et al. (2002) due to the lower signal-to-noise ratios in their data sets.

In Table 4 we give a list of the variable stars we have observed. The columns in Table 4 contain the variable name, 


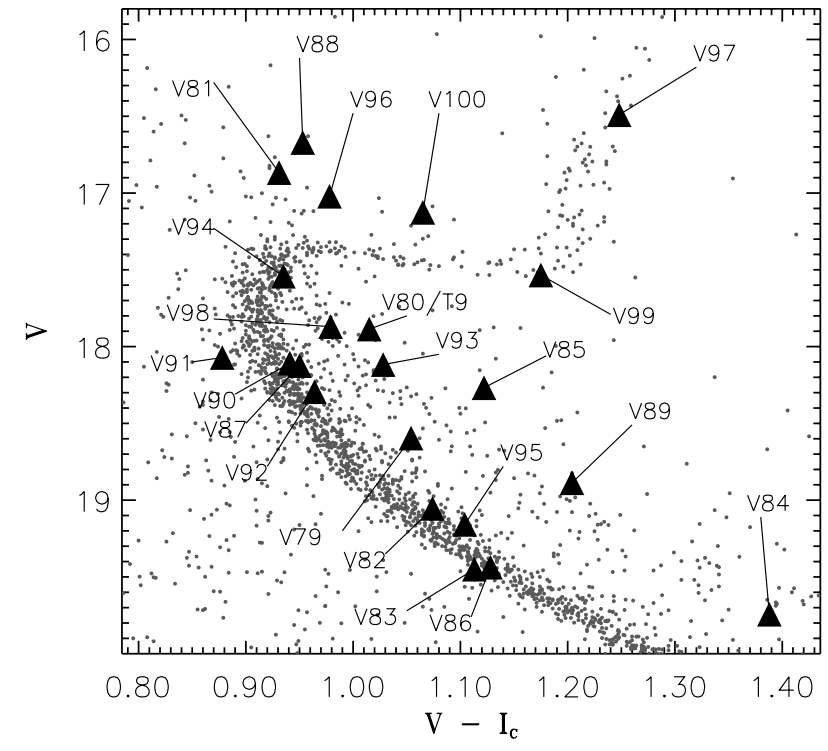

Fig. 5. Colour-magnitude diagram of NGC 6791 with standard $V$ and $V-I_{\mathrm{c}}$ magnitudes from Stetson et al. (2003). The 22 newly discovered variables have been marked. Only the stars with the best photometry are plotted to emphasize the location of the main sequence, the turnoff, and the red giant branch.

the ID number, right ascension, declination, period, the approximate amplitude in the $V$ filter, and the variable type. The information in Cols. 2-4 is from Stetson et al. (2003).

We have determined the period of each star by using the method of PERIOD98 (Sperl 1998). For some of the new longperiod variables the time series is not long enough or the amplitude is too small to give a safe estimate of the period. In these cases we have only given an approximate period (indicated by the " " symbol). Except for some of the long period variables, our period estimates agree with previously published results.

In Fig. 6 we show the phased light curves of some of the known variables and in Fig. 7 we present some the new variables. The ordinate gives the change in magnitude in the $V$ filter and the phase is on the abscissa.

The MOCHO1 and SMR95 data are not plotted in Figs. 6 and 7 if the amplitude of the variable is comparable to the noise level in these data sets.

An interesting eclipsing binary that was previously known is V9, which was observed in all three data sets. It is classified as an RS Canum Venaticorum type star (RS CVn, see e.g., Hall 1972). The light curves in the $V$ (left plot) and $I$ filters are shown in Fig. 8 where the "+" symbols are used for the NOTO1 data, the filled grey symbols are the MOCHO1 data, while the squares are the SMR95 data. The light curve of V9 is characterized by a deep eclipse which is modulated by a "distortion wave" probably due to dark spots on the surface of one of the stars in the binary pair. It is interesting to note that while the phase of the eclipse is unchanged the modulation wave has shifted phase from 1995 (SMR95) to 2002 (NOTO1 and $\mathrm{MOCHO1}$ ). The shape of the modulation wave seems to be unchanged, as was also noted by Mochejska et al. (2002).
We finally note that the light curves presented here will be available from the Canadian Astronomy Data Centre (Stetson et al. 2003).

\section{Three eclipsing binary stars in NGC 6791}

In Fig. 9 we show the light curves of three detached eclipsing binaries. In each panel $t_{0}$ is the time HJD-2452000 for the tick-mark corresponding to 0.0 . The black and grey points are $V$ and $I$ measurements, while open circles are $R$ measurements from $\mathrm{MOCHO1.}$

For each of the eclipsing binaries V20, V60, and V79 we show the light curves from five nights, i.e., the nights on which the eclipses occur and one or more nights without eclipses for comparison.

The eclipses of V20 and V60 were noted before by Rucinski (1996) and Mochejska et al. (2002), but now for the first time we have enough time coverage to be able to constrain the periods. From the difference in time between the eclipses we find periods of $P_{\text {bin }}=14.470 \pm 0.001$ and $7.510 \pm 0.005$ days for V20 and V60, respectively. We note that V60 is variable with an amplitude of $0.015 \mathrm{mag}$ in $V$ at a period of $P_{\mathrm{var}} \simeq 7.1 \pm 0.2$ days.

\subsection{The detached binary V20}

We have been granted observing time to obtain more detailed photometric coverage of the primary and secondary eclipses of V20, and we plan to carry out spectroscopic measurements to obtain the radial velocity curve. With these observations it will be possible to constrain the masses, radii, and luminosities of the binary components of this system.

As will be demonstrated in Sect. 7.2, the primary component of V20 is very close to the turnoff. From the knowledge of the mass of the primary component, we expect to be able to determine the age of NGC 6791 with good accuracy. This can be done from the relation between age and mass of the turnoff stars which will rely on theoretical isochrones.

This has recently been done for the globular cluster $\omega$ Cen by Thompson et al. (2001) and Kaluzny et al. (2002) for eclipsing stars around the turnoff. Their masses could be determined with an accuracy of just $1 \%$, including systematic errors. In this case one of the systems is right after the turnoff and Kaluzny et al. (2002) were able to constrain the position of the theoretical isochrones and in turn estimate the age of $\omega$ Cen to within $0.6 \mathrm{Gyr}$ - at least a factor of two better than any previous age determination for a globular cluster.

\subsection{Estimating the parameters of the detached binary V20}

In Fig. 10 the colour-magnitude diagram of NGC 6791 is shown. The location of the eclipsing binary V20 is shown with a star symbol above the turnoff region. In the following we will describe how we have determined the parameters of the two components that make up the binary V20.

We have fitted a fiducial locus to the turnoff region shown as the solid "primary" curve in Fig. 10, and in the following 
Table 4. The variables in the NOTO1 field. The first column contains the variable name, where the new variables are printed with italics (V79V100). Columns 2-4 contain ID, right ascension, and declination from Stetson et al. (2003). Columns 5 and 6 are the periods and amplitudes (in the $V$ filter) we have determined. The last column gives the variable star type. The positions of the variables in the colour-magnitude diagram are given in Figs. 3 and 5 while the phased light curves of some of the stars are shown in Figs. 6, 7, and 8.

\begin{tabular}{|c|c|c|c|c|c|c|}
\hline Name & ID & $\alpha_{2000.0}$ & $\delta_{2000.0}$ & $P$ [days] & $A[\mathrm{mag}]$ & Var. type \\
\hline V1 & 6271 & $19^{\mathrm{h}} 20^{\mathrm{m}} 47^{\mathrm{s}} .61$ & $+37^{\circ} 44^{\prime} 32^{\prime \prime} .0$ & 0.2676766 & 0.194 & EB, close \\
\hline V4 & 8576 & $19^{\mathrm{h}} 20^{\mathrm{m}} 54^{\mathrm{s}} .22$ & $+37^{\circ} 48^{\prime} 23^{\prime \prime} .8$ & 0.325667 & 0.046 & $\mathrm{~EB}$, close \\
\hline V5 & 5883 & $19^{\mathrm{h}} 20^{\mathrm{m}} 46^{\mathrm{s}} .53$ & $+37^{\circ} 48^{\prime} 47^{\prime \prime} .9$ & 0.312666 & 0.017 & $\mathrm{~EB}$, close \\
\hline V6 & 11376 & $19^{\mathrm{h}} 21^{\mathrm{m}} 02^{\mathrm{s}} .72$ & $+37^{\circ} 48^{\prime} 49^{\prime}{ }^{\prime} 1$ & 0.279062 & 0.042 & $\mathrm{~EB}$, close \\
\hline V9 & 6371 & $19^{\mathrm{h}} 20^{\mathrm{m}} 47^{\mathrm{s}} .88$ & $+37^{\circ} 46^{\prime} 37^{\prime \prime} .4$ & 3.19174 & $0.040^{a}$ & $\mathrm{~EB}$, close \\
\hline V16 & 12695 & $19^{\mathrm{h}} 21^{\mathrm{m}} 07^{\mathrm{s}} .59$ & $+37^{\circ} 48^{\prime} 09^{\prime} 6$ & 4.53 & 0.026 & $\mathrm{~EB}$, close \\
\hline V31 & 11307 & $19^{\mathrm{h}} 21^{\mathrm{m}} 02^{\mathrm{s}} .47$ & $+37^{\circ} 47^{\prime} 09^{\prime} 3$ & 3.34154 & 0.015 & $\mathrm{~EB}$, close \\
\hline V33 & 3886 & $19^{\mathrm{h}} 20^{\mathrm{m}} 39^{\mathrm{s}} .81$ & $+37^{\circ} 43^{\prime} 54^{\prime \prime} .4$ & 2.35944 & 0.040 & $\mathrm{~EB}$, close \\
\hline V38 & 11652 & $19^{\mathrm{h}} 21^{\mathrm{m}} 03^{\mathrm{s}} .69$ & $+37^{\circ} 46^{\prime} 05^{\prime \prime} .9$ & 3.845 & 0.023 & $\mathrm{~EB}$, close \\
\hline V20 & 8600 & $19^{\mathrm{h}} 20^{\mathrm{m}} 54^{\mathrm{s}} .30$ & $+37^{\circ} 45^{\prime} 34^{\prime \prime} .7$ & 14.4698 & $0.3^{a}$ & $\mathrm{~EB}$, detached \\
\hline V60 & 10746 & $19^{\mathrm{h}} 21^{\mathrm{m}} 00^{\mathrm{s}} .70$ & $+37^{\circ} 45^{\prime} 45^{\prime \prime} .1$ & $7.510 / 7.1^{b}$ & $0.4 / 0.015^{b}$ & EB, detached \\
\hline$V 79$ & 8943 & $19^{\mathrm{h}} 20^{\mathrm{m}} 55^{\mathrm{s}} .21$ & $+37^{\circ} 46^{\prime} 39^{\prime} .7$ & $\sim 9.0038$ & $0.15^{a}$ & EB, detached \\
\hline$V 80^{c}$ & 12390 & $19^{\mathrm{h}} 21^{\mathrm{m}} 06^{\mathrm{s}} .48$ & $+37^{\circ} 47^{\prime} 27^{\prime \prime} .8$ & 4.447 & 0.014 & EB? \\
\hline V14 & 7637 & $19^{\mathrm{h}} 20^{\mathrm{m}} 51^{\mathrm{s}} .67$ & $+37^{\circ} 45^{\prime} 24^{\prime \prime} .8$ & $5.4-5.8$ & 0.028 & Periodic Var. \\
\hline V17 & 3626 & $19^{\mathrm{h}} 20^{\mathrm{m}} 38^{\mathrm{s}} .88$ & $+37^{\circ} 49^{\prime} 04^{\prime \prime} .6$ & 6.42566 & 0.036 & Periodic Var. \\
\hline V41 & 7397 & $19^{\mathrm{h}} 20^{\mathrm{m}} 50^{\mathrm{s}} .97$ & $+37^{\circ} 48^{\prime} 24^{\prime \prime} .8$ & 0.4807278 & 0.039 & Periodic Var. \\
\hline V53 & 10798 & $19^{\mathrm{h}} 21^{\mathrm{m}} 00^{\mathrm{s}} .84$ & $+37^{\circ} 44^{\prime} 35^{\prime \prime} .4$ & 7.815 & 0.039 & Periodic Var. \\
\hline$V 81$ & 3268 & $19^{\mathrm{h}} 20^{\mathrm{m}} 49^{\mathrm{s}} .65$ & $+37^{\circ} 48^{\prime} 08^{\prime \prime} .7$ & 7.58 & 0.008 & Periodic Var. \\
\hline$V 82$ & 3857 & $19^{\mathrm{h}} 20^{\mathrm{m}} 39^{\mathrm{s}} .71$ & $+37^{\circ} 47^{\prime} 36^{\prime \prime} .1$ & 7.58886 & 0.010 & Periodic Var. \\
\hline$V 83$ & 5848 & $19^{\mathrm{h}} 20^{\mathrm{m}} 46^{\mathrm{s}} 40$ & $+37^{\circ} 44^{\prime} 14^{\prime \prime} .1$ & 7.014 & 0.037 & Periodic Var. \\
\hline$V 84$ & 6305 & $19^{\mathrm{h}} 20^{\mathrm{m}} 47^{\mathrm{s}} .71$ & $+37^{\circ} 44^{\prime} 58^{\prime \prime} .2$ & 1.6333 & 0.03 & Periodic Var. \\
\hline V85 & 7011 & $19^{\mathrm{h}} 20^{\mathrm{m}} 49^{\mathrm{s}} .86$ & $+37^{\circ} 45^{\prime} 50^{\prime} .9$ & 4.100 & 0.007 & Periodic Var., EB? \\
\hline$V 86$ & 7099 & $19^{\mathrm{h}} 20^{\mathrm{m}} 50^{\mathrm{s}} 13$ & $+37^{\circ} 48^{\prime} 31^{\prime \prime} .7$ & 7.39 & 0.011 & Periodic Var. \\
\hline$V 87$ & 8033 & $19^{\mathrm{h}} 20^{\mathrm{m}} 52^{\mathrm{s}} .78$ & $+37^{\circ} 44^{\prime} 58^{\prime \prime} .8$ & 6.971 & 0.006 & Periodic Var. \\
\hline$V 88$ & 8088 & $19^{\mathrm{h}} 20^{\mathrm{m}} 52^{\mathrm{s}} .91$ & $+37^{\circ} 46^{\prime} 36^{\prime \prime} .9$ & 6.91 & 0.008 & Periodic Var. \\
\hline V89 & 9432 & $19^{\mathrm{h}} 20^{\mathrm{m}} 56.64$ & $+37^{\circ} 46^{\prime} 36^{\prime \prime} .2$ & 5.7617 & 0.028 & Periodic Var. \\
\hline$V 90$ & 10169 & $19^{\mathrm{h}} 20^{\mathrm{m}} 58^{\mathrm{s}} .86$ & $+37^{\circ} 44^{\prime} 47^{\prime \prime} .2$ & 5.71 & 0.008 & Periodic Var. \\
\hline$V 91$ & 10699 & $19^{\mathrm{h}} 21^{\mathrm{m}} 00^{\mathrm{s}} .54$ & $+37^{\circ} 48^{\prime} 40^{\prime} .7$ & 5.13 & 0.011 & Periodic Var. \\
\hline V92 & 11380 & $19^{\mathrm{h}} 21^{\mathrm{m}} 02^{\mathrm{s}} .73$ & $+37^{\circ} 46^{\prime} 00^{\prime} .8$ & 7.24 & 0.008 & Periodic Var. \\
\hline V93 & 12049 & $19^{\mathrm{h}} 21^{\mathrm{m}} 05^{\mathrm{s}} .23$ & $+37^{\circ} 47^{\prime} 08^{\prime \prime} .5$ & 0.94928 & 0.007 & Periodic Var. \\
\hline B7 & 12652 & $19^{\mathrm{h}} 21^{\mathrm{m}} 07^{\mathrm{s}} .40$ & $+37^{\circ} 47^{\prime} 56^{\prime \prime} .5$ & $\sim 13.3$ & 0.15 & Long period \\
\hline V62 & 11475 & $19^{\mathrm{h}} 21^{\mathrm{m}} 03^{\mathrm{s}} .06$ & $+37^{\circ} 43^{\prime} 51^{\prime \prime} .8$ & $\sim 17$ & 0.027 & Long period \\
\hline V65 & 7920 & $19^{\mathrm{h}} 20^{\mathrm{m}} 52^{\mathrm{s}} .47$ & $+37^{\circ} 47^{\prime} 30^{\prime} .5$ & 8.613 & 0.008 & Long period \\
\hline V67 & 11645 & $19^{\mathrm{h}} 21^{\mathrm{m}} 03^{\mathrm{s}} .67$ & $+37^{\circ} 48^{\prime} 03^{\prime \prime} .7$ & $\sim 10$ & 0.05 & Long period \\
\hline V73 & 8108 & $19^{\mathrm{h}} 20^{\mathrm{m}} 52^{\mathrm{s}} .97$ & $+37^{\circ} 46^{\prime} 52^{\prime \prime} .5$ & $\sim 20.9$ & 0.02 & Long Period \\
\hline$V 94$ & 4640 & $19^{\mathrm{h}} 20^{\mathrm{m}} 42^{\mathrm{s}} .50$ & $+37^{\circ} 44^{\prime} 36^{\prime \prime} .9$ & $\sim 15.57$ & 0.01 & Long period \\
\hline$V 95$ & 4805 & $19^{\mathrm{h}} 20^{\mathrm{m}} 43^{\mathrm{s}} .05$ & $+37^{\circ} 47^{\prime} 32^{\prime \prime} .5$ & 9.477 & 0.012 & Long period \\
\hline$V 96$ & 5485 & $19^{\mathrm{h}} 20^{\mathrm{m}} 45^{\mathrm{s}} .26$ & $+37^{\circ} 45^{\prime} 48^{\prime \prime} .8$ & 9.307 & 0.012 & Long Period \\
\hline V97 & 6777 & $19^{\mathrm{h}} 20^{\mathrm{m}} 49^{\mathrm{s}} .17$ & $+37^{\circ} 49^{\prime} 14^{\prime \prime} .8$ & 9.563 & 0.013 & Long period \\
\hline V98 & 9353 & $19^{\mathrm{h}} 20^{\mathrm{m}} 56^{\mathrm{s}} .42$ & $+37^{\circ} 45^{\prime} 38^{\prime \prime} .4$ & $\sim 8.99$ & 0.01 & Long period \\
\hline$V 99$ & 9568 & $19^{\mathrm{h}} 20^{\mathrm{m}} 57^{\mathrm{s}} .06$ & $+37^{\circ} 48^{\prime} 12^{\prime \prime} .2$ & $\sim 10$ & 0.003 & Long period \\
\hline$V 100$ & 11111 & $19^{\mathrm{h}} 21^{\mathrm{m}} 01^{\mathrm{s}} .81$ & $+37^{\circ} 45^{\prime} 42^{\prime \prime} .0$ & 12.5215 & 0.01 & Long period \\
\hline
\end{tabular}

${ }^{a}$ For the eclipsing binaries V9, V20, and $V 79$ the depth of the primary eclipse in the $V$ filter is given.

${ }^{b}$ V60 is variable with a low amplitude and a period of $P \sim 7.1$ days while the period estimated from the secondary and primary eclipses is $7.510 \pm 0.005$ days. The primary eclipse depth is 0.4 mag in $V$.

${ }^{c}$ V80 was detected in our search for transit-like events and is also given the name $T 9$ (cf Fig. 4).

Furthermore, $V 80$ is variable with a low amplitude. 

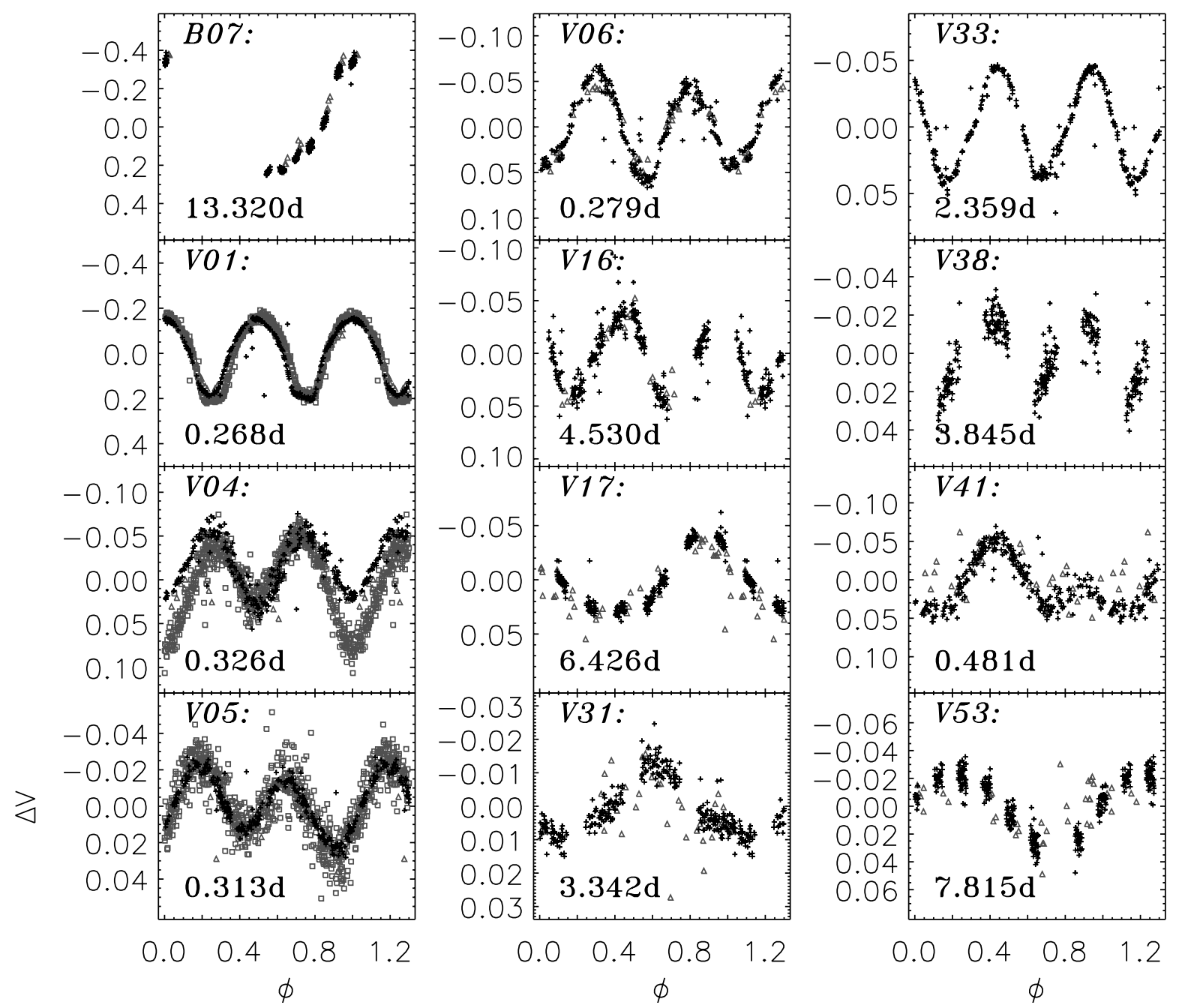

Fig. 6. Phased light curves in the $V$ filter for some of the known variables in NGC 6791. In most panels only the NOT01 data (+ symbols) are plotted due to the much lower noise level compared to MOCHO1 (triangle symbols) and SMR95 (box symbols). Notice the change in the light curve of V4.

we assume the primary component will be on this curve. For each point on the "primary" curve we calculate what the magnitude and colour of the secondary component should be, while requiring that the combined colour and luminosity of the primary and secondary component must yield the observed position of V20. The inferred position of the secondary component is shown as the dashed "secondary" curve.

For three positions on the "primary" curve we have marked the corresponding position on the "secondary" curve: at the turnoff (TO), on the main sequence (MS), and the point where the main sequence and the "secondary" curve intersect (Bin). The latter is the best fit of the location of the two components making up the binary V20. Assuming that this inference is correct, their colours are determined to within less than $\pm 0.01 \mathrm{mag}$; these are given in Table 5 . We will now use these colour estimates to infer the properties of the components of V20.

We have used the calibration of $T_{\text {eff }}$ vs. $(V-I)_{J}$ for main sequence stars with solar metallicity by Alonso et al. (1996). The calibration of Alonso et al. (1996) refer to Johnson $I_{J}$ magnitudes, and so we have used the calibration of Fernie (1983) to convert from the Kron-Cousins system we have used, i.e., $(V-I)_{J}=-0.005+1.273(V-I)_{C}$. For the interstellar reddening we use $E(B-V)=0.105 \pm 0.025$ (Chaboyer et al. 1999) and using Taylor (1986) we find $E(V-I)=0.13 \pm 0.03$. We note that the error given on $E(B-V)$ does not include systematic errors which could very likely be of the order $\sigma(E(B-V)) \simeq 0.05$. However, the analysis of the colour-magnitude diagram of the cluster by Stetson et al. (2003) suggests a reddening $E(B-$ $V) \sim 0.09$, entirely consistent with the Chaboyer value that we employ.

From these numbers we can determine the de-reddened magnitudes and colours and thus estimate the $T_{\text {eff' }} \mathrm{s}$ of the two components of V20 to within $200 \mathrm{~K}$, including the systematic uncertainty in $E(B-V)$. The results are given in Table 5 . We obtain the bolometric correction from Bessell et al. (1998) for atmospheric models with solar metallicity and no overshooting. We can then determine the relative luminosities of the stars using $M_{\mathrm{bol} ; \mathrm{p}}-M_{\mathrm{bol} ; \mathrm{s}}=\left(V_{\mathrm{p}}+B C_{\mathrm{p}}\right)-\left(V_{\mathrm{s}}+B C_{\mathrm{s}}\right)=-2.84 \pm 0.14$ which is equal to $-2.5 \log \left(L_{\mathrm{p}} / L_{\mathrm{s}}\right)$, and hence the luminosity ratio is $L_{\mathrm{p}} / L_{\mathrm{s}}=13.7 \pm 1.8$. When using $L \propto R^{2} T_{\text {eff }}{ }^{4}$ we find a radius ratio of $R_{\mathrm{s}} / R_{\mathrm{p}}=0.41 \pm 0.08$. 

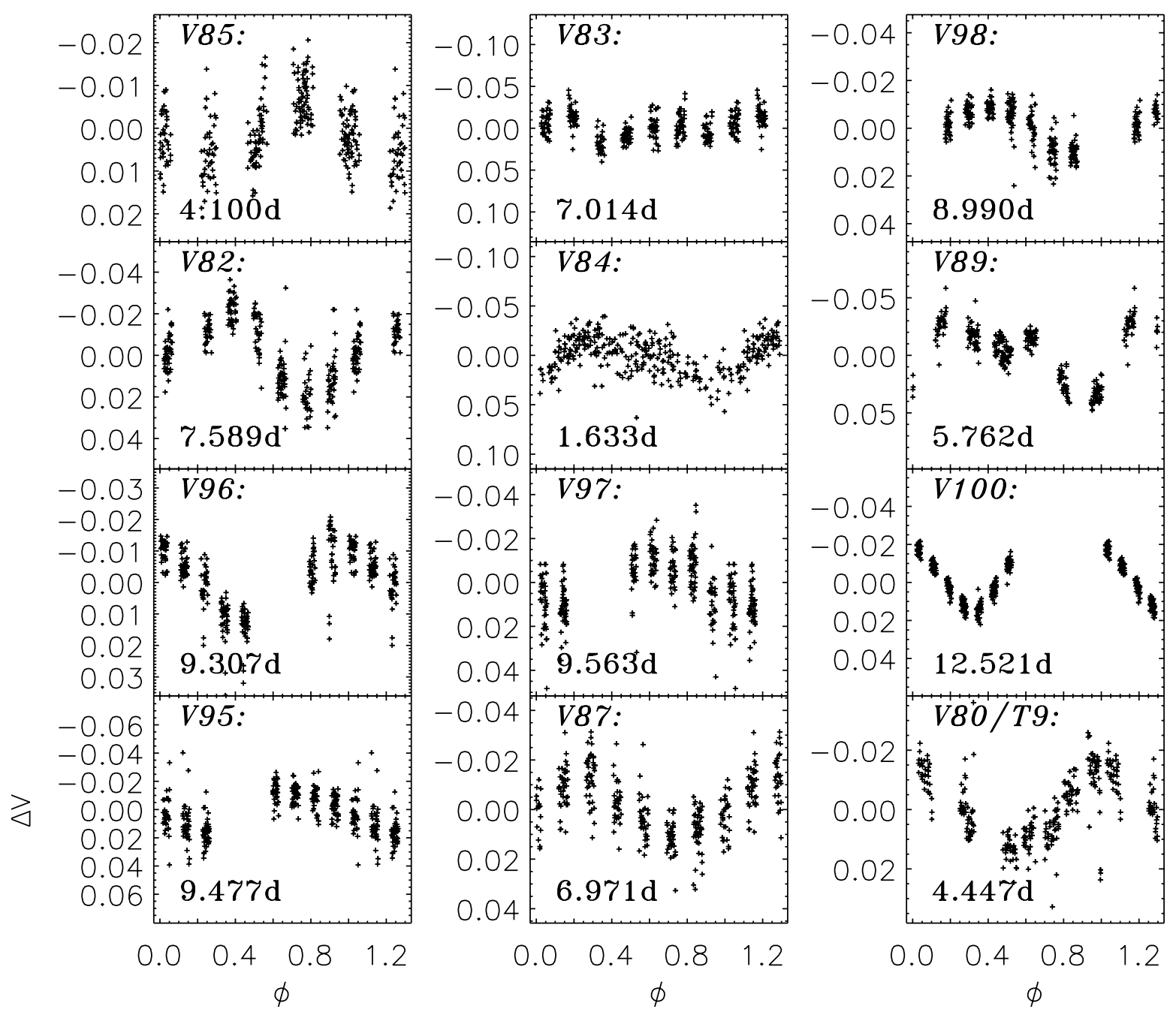

Fig. 7. Phased light curves of some of the new low-amplitude variables. Only the NOTO1 data are plotted. The ID number is given in the top left corner of each panel and correspond to Stetson et al. (2003). The period (in days) is given in the lower left corner. More details are given in Table 4.

We have also estimated the relative luminosities of the binary components by modelling the observed light curve of V20 following the method outlined in Clausen et al. (2003). We assumed $T_{\text {eff }}=5200 \mathrm{~K}$ for the primary component and from the modelling of the light curves using only the light curve in $V$ we find $T_{\text {eff }}=4400 \pm 250 \mathrm{~K}$ for the secondary component. The large error in $T_{\text {eff }}$ is mainly due to the depth of the secondary eclipse being different by $\simeq 0.02 \mathrm{mag}$ in the SMR95 and NOTO1 data set. From the light-curve modelling we also derive the radius ratio to be $R_{\mathrm{s}} / R_{\mathrm{p}}=0.55 \pm 0.1$. Both $T_{\text {eff }}$ and the radius ratio are in good agreement with what we have found by the two methods described here.

Obviously, much more detailed photometric coverage of the primary and secondary eclipses of V20 are needed to further constrain the properties of the two components. As already mentioned, we need spectroscopic observations of the complete orbit to be able to constrain the masses of the components.

\section{Conclusion and future prospects}

We have described the search for planet transits in the open cluster NGC 6791 from seven nights of observations with the
Nordic Optical Telescope. The photometric precision is adequate for detecting transits by close-in Jupiter-sized planets. We have found ten stars with transit-like events in the light curves, but we argue that most of these are probably not caused by a transiting giant planet. The observed transit events may be due to instrumental effects (bad columns, offset of the telescope) and in one case there is evidence that the star (T9) belongs to a binary system. For the remaining three transit-like events the precision ( $3 \mathrm{mmag}$ ) is not good enough compared to the very shallow transit depth $(\sim 10 \mathrm{mmag})$ to tell whether the transits are really caused by giant planets, e.g., to see whether the light curves are flat during the transit. To confirm the validity of these three planetary transit candidates the observation of multiple transits with higher precision are required.

In July 2002 Piotto, Stetson et al. have carried out a more ambitious multi-site campaign on NGC 6791 to search for planet transits. We have obtained observations on eight at least partially clear nights of a ten-night run on the CFHT $3.6 \mathrm{~m}$ telescope in Hawaii during 2002 July 2-12. In the same period of time observations from Mexico and Italy were also carried out. The weather was not optimal during the multi-site campaign on NGC 6791 in 2002, but we have a higher signal-to-noise ratio 


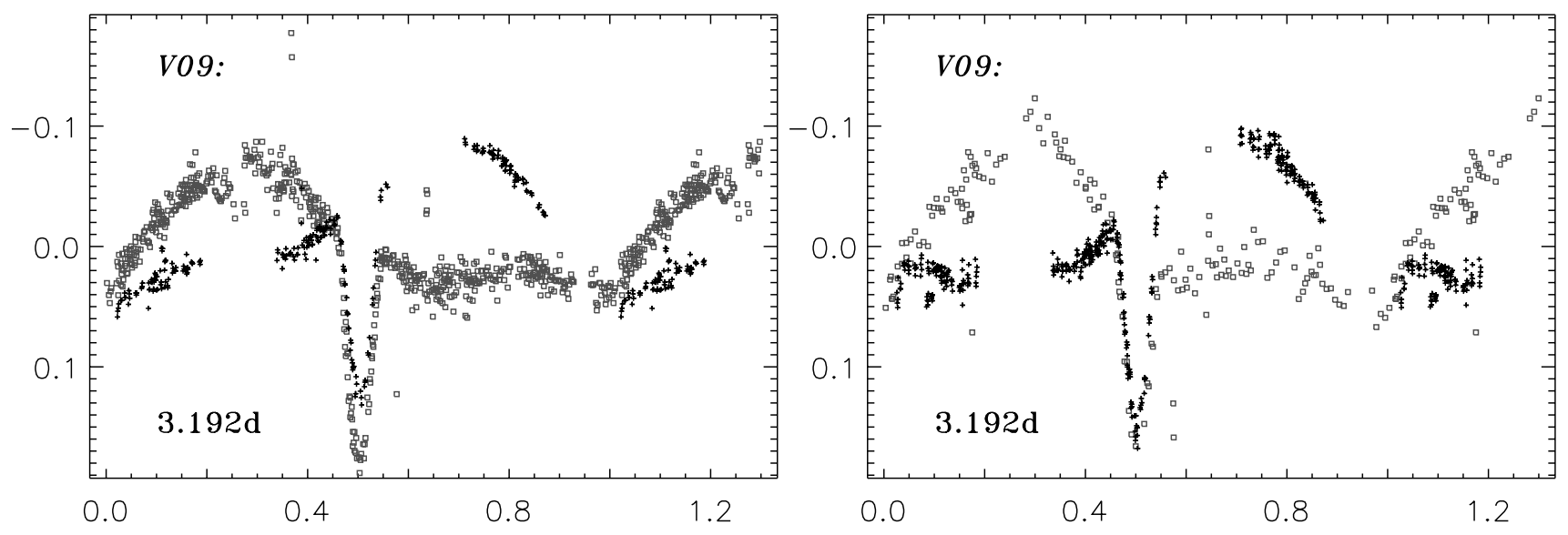

Fig. 8. Phased light of the RS CV-type variable V9 in NGC 6791 in the $V$ (left plot) and $I$ filter (right plot). The black + symbols are data from NOTO1 while the grey symbols are from either SMR95 (box symbol) or MOCHO1 (filled grey symbols) The MOCHO1 data are not plotted in the right plot since Mochejska et al. (2002) did not observe in $I$.
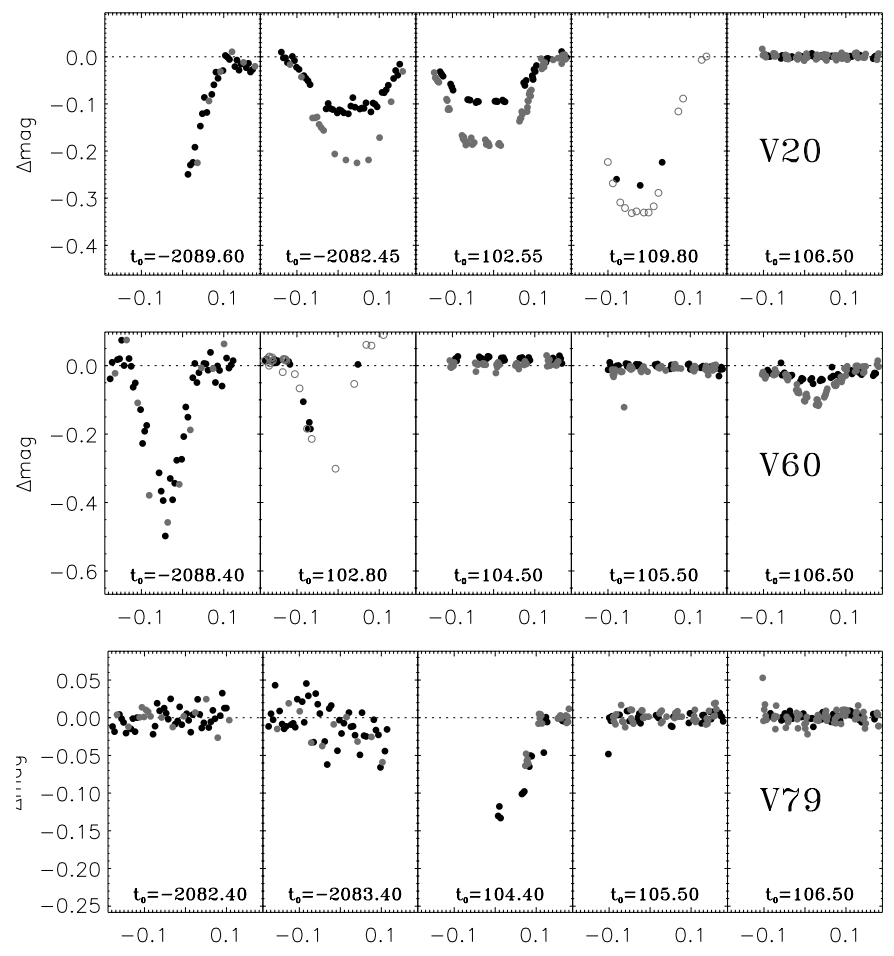

Fig. 9. Light curves of three eclipsing binary stars. The time corresponding to 0.0 on the abscissa is HJD-2 452000 and is given at the bottom of each panel as $t_{0}$. One or more nights without eclipses are shown for comparison. Black and grey symbols are $V$ and $I$ measurements, while the open circles are $R$ measurements from MOCHO1.

compared to the NOTO1 data and also a higher duty cycle over 10 nights. This will improve the statistics for detecting multiple transits. The reduction of data from the 2002 campaign data is now under way.

In addition to our own data from NOTO1 we have used data from two previous photometric campaigns. The photometric precision of these other data sets is not high enough to confirm the shallow transits we have found. We have used the complete data set to redetermine the periods of the 20 known variables

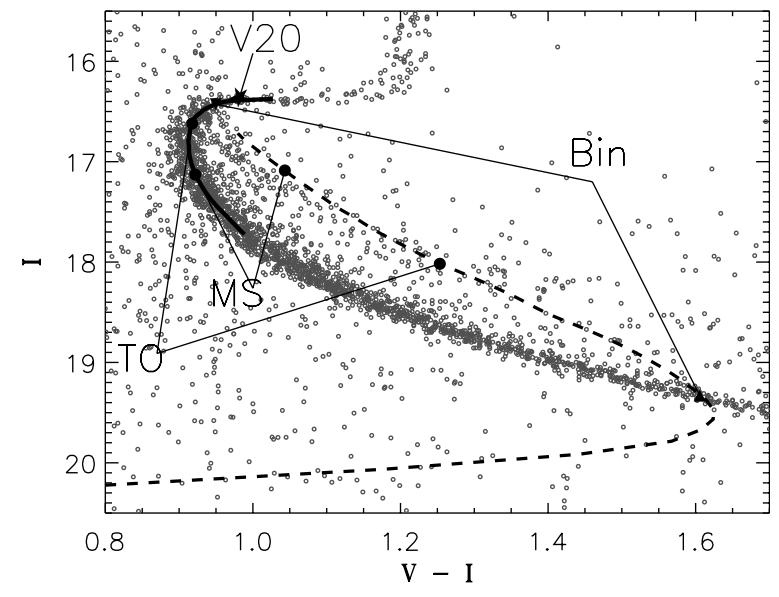

Fig. 10. The $I$ vs. $V-I$ colour-magnitude diagram of NGC 6791 on the standard system. The location of the eclipsing binary V20 above the turnoff is shown with a star symbol. To find the possible positions of the two components of the binary V20, we assume that the primary star is on the solid black curve and calculate the corresponding colour and magnitude for the secondary component which is given as the dashed line. For three points we have shown the location of the primary and secondary star, i.e., for the primary star being at the turnoff (TO), further down the main sequence (MS), and the point where the dashed curve intersects the main sequence (Bin).

in the NOTO1 field. Furthermore, we have discovered 22 new long-period stars with low amplitudes.

We have determined the periods of two detached eclipsing binaries, V20 and V60. From the position of V20 in the colourmagnitude diagram we are able to constrain the temperatures of the binary components. This is in good agreement with results from the modelling of the primary and secondary eclipses. We find that the primary component is located just after the turnoff, while the secondary component is a much fainter main sequence star. We plan to make thorough photometric and spectroscopic observations of V20 to constrain the masses of the binary components. From the mass of the primary star we can determine the age of NGC 6791 from a comparison with the 
Table 5. The derived parameters for the two components of the binary star V20. We found the colours from Fig. 10, and in the text we explain how $T_{\text {eff }}$ was determined. The estimated errors are given in parenthesis.

\begin{tabular}{c|cccc|c}
\hline \hline Component & $V$ & $V-I$ & $(V-I)_{0}$ & $T_{\text {eff }}$ & \multicolumn{1}{|c|}{$B C_{V}$} \\
\hline Primary & $17.38(1)$ & $0.95(1)$ & $0.82(6)$ & $5200(200)$ & $-0.20(5)$ \\
Secondary & $20.94(1)$ & $1.61(1)$ & $1.48(6)$ & $4070(200)$ & $-0.96(10)$ \\
\hline
\end{tabular}

calculated turnoff mass for isochrones of different ages. This will give an age estimate independent of interstellar reddening.

\section{References}

Alard, C., \& Lupton, R. H. 1998, ApJ, 503, 325

Alonso, A., Arribas, S., \& Martinez-Roger, C. 1996, A\&A, 313, 873

Bessell, M. S., Castelli, F., \& Plez, B. 1998, A\&A, 333, 231

Burke, C. J., DePoy, D. L., Gaudi, B. S., Marshall, J. L., \& Pogge, R. W. 2002 [astro-ph/0208305]

Burrows, A., Guillot, T., Hubbard, W. B., et al. 2000, ApJ, 534, L97

Butler, R. P., Marcy, G. W., Fischer, D. A., et al. 2002, ed., A. Penny, et al., IAU Symp. 202, ASP Conf. Ser., in press

Chaboyer, B., Green, E. M., \& Liebert, J. 1999, AJ, 117, 1360

Charbonneau, D., Brown, T. M., Latham, D. W., \& Mayor, M., 2000, ApJ, 529, L45

Clausen, J. V., Storm, J., Larsen, S. S., \& Giménez, A. 2003, A\&A, 402, 509

Cochran, W. D., Hatzes, A. P., \& Paulson, D. B. 2002, AJ, 124, 565

Dreizler, S., Rauch, T., Hauschildt, P., et al. 2002, A\&A, 391, L17

Fernie, J. D. 1983, PASP, 95, 782

Gonzalez, G., Laws, C., Tyagi, S., \& Reddy, B. E. 2001, AJ, 121, 432

Grundahl, F., \& Sørensen, A. N. 1996, A\&AS, 116, 367

Hall, D. S. 1972, PASP, 84, 323

Jenkins, J., Doyle, L., \& Culler, D. 1996, Icarus, 119, 244

Kaluzny, J. 2003, Acta Astron., 53, 51

Kaluzny, J., Thompson, I., Krzeminski, W., et al. 2002, ASP Conf. Proc., 265, 155
Kjeldsen, H., \& Frandsen, S. 1992, PASP, 104, 413

Laughlin, G. 2000, ApJ, 545, 1064

Mallén-Ornelas, G., Seager, S., Yee, H. K. C., et al. 2003, ApJ, 582, 1123

Mayor, M., \& Queloz, D. 1995, Nature, 378, 355

Mochejska, B. J., Stanek, K. Z., Sasselov, D. D., \& Szentgyorgyi, A. H. 2002, AJ, 123, 3460

Mochejska, B. J., Stanek, K. Z., \& Kaluzny, J. 2003, AJ, 125, 3175

Rocha-Pinto, H. J., \& Maciel, W. J. 1996, MNRAS, 279, 447

Rucinski, S. M., Kaluzny, J., \& Hilditch, R. W. 1996, MNRAS, 282, 705

Salaris, M., \& Weiss, A. 1998, A\&A, 335, 943

Sperl, M. 1998, Comm. in Asteroseismology, 111, 1

Stetson, P. B. 1987, PASP, 99, 191

Stetson, P. B. 1990, PASP, 102, 932

Stetson, P. B. 1994, PASP, 106, 250

Stetson, P. B. 1996, PASP, 108, 851

Stetson, P. B., Saha, A., Ferrarese, L., et al. 1998, ApJ, 508, 491

Stetson, P. B. 2000, PASP, 112, 925

Stetson, P. B., Bruntt, H., \& Grundahl, F. 2003, PASP, 115, 413

Taylor, B. J. 1986, ApJS, 60, 577

Taylor, B. J. 2001, A\&A, 377, 473

Thompson, I. B., Kaluzny, J., Pych, W., et al. 2001, AJ, 121, 3089

Tingley, B. 2003, A\&A, 403, 329

Udalski, A., Paczynski, B., Zebrun, K., et al. 2002a, Acta Astron., 52, 1

Udalski, A., Zebrun, K., Szymanski, M., et al. 2002b, Acta Astron., 52,115

Wozniak, P. R., Udalski, A., Szymanski, M., et al. 2002, Acta Astron., 52,129 